\section{Don't defer, refer!}

Many patients are currently seeing increased waiting times for routine treatment as practices adapt to challenging circumstances. If you receive a patient with urgent and/ or complex needs outside that you need support to treat, consider referring them to the Centre for Oral-Maxillofacial and Dental Implant Reconstruction.

The advanced centre offers a variety of treatment for clinical indications including surgical extractions and oral surgery for failing implants, severely atrophic jaws and nerve damage. The highly experienced team is led by Professor Cemal Ucer - a Specialist Oral Surgeon who is renowned throughout the profession for his expertise in the field. The practice also utilises state-of-the-art technologies, materials and systems that facilitate the evidence-based treatment protocols.

In some situations, you don't want to defer patient care - refer them to the Centre for Oral-Maxillofacial and Dental Implant Reconstruction.

Contact Professor Ucer at ice@ucer. uk or Mel Hay at mel@mdic.co 01612 371842 .

\section{Speed, accuracy, versatility}

Have you ever been frustrated with an impression material because it doesn't give you accurate results?

With Imprint 4 VPS Impression Material from $3 \mathrm{M}$, this is no longer a concern.

Imprint 4 VPS impression material is specifically designed with unique chemistry which means the setting time doesn't start until the end of the working time. That means you'll have a sufficient amount of time to load and seat the tray no stressful race against the clock.

In addition, Imprint 4 VPS impression material is super-hydrophilic and displaces moisture right from the start in the unset state. This allows the material to reproduce accurate details even in a moist environment - giving you ultimate precision and confidence.

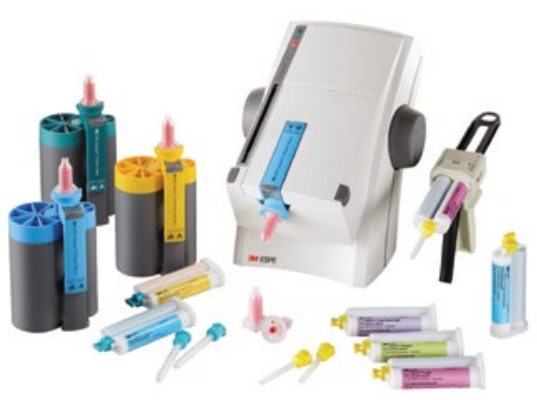

It's no wonder that Imprint 4 VPS impression material from $3 \mathrm{M}$ has been awarded 'Best VPS Impression Material' by The Dental Advisor for seven consecutive years.

For more information, call 0845873 4066 or visit www. 3 m.co.uk/dental. $3 \mathrm{M}$ representatives remain contactable by phone or via video conferencing.

$3 \mathrm{M}$ and Imprint 4 are trademarks of the 3M Company.

\section{Safeguard your livelihood}

It has never been more important to safeguard your livelihood and standard of living than right now with effective income protection.

There are many elements to consider, from exclusions to protection from inflation, guaranteed or reviewable rates and what criteria needs to be met to make a claim. It's vital to read the fine print to ensure that you and your family will be kept safe in the event that you are unable to work due to illness, accident or injury.

The expert team at money4dentists can help you review an existing policy or arrange a new solution that affords total peace of mind. They know what to look for and how to tailor your policy for your specific needs. Give them a call today to find out more.

For more information call 0845345 5060, email info@money4dentists.com or visit www.money4dentists.com.

\title{
Getting back to in-person learning
}

When it is safe to do so, the benefits of hands-on training and in-person learning cannot be beaten. Don't miss your chance to develop your skills at the British Dental Conference and Dentistry Show 2021 - on Friday 25 and Saturday 26 June - where you will have access to hours of enhanced CPD and live demonstrations.

There will be conference streams dedicated to each area of the profession, with world-class speakers sharing their expertise on a wide range of topics. The extensive trade floor will also host a dynamic selection of dental manufacturers and suppliers with innovative technologies and materials to discover

Delegates will be able to interact with thousands of colleagues, friends, product specialists and renowned figures at the very forefront of their respective fields.
To get back to quality in-person learning, don't miss the British Dental Conference and Dentistry Show 2021!

The British Dental Conference and Dentistry Show 2021 will be held on Friday 25 and Saturday 26 June, Birmingham NEC, co-located with DTS.

For more information, visit www. thedentistryshow.co.uk, call 02073485270 or email dentistry@closerstillmedia.com.

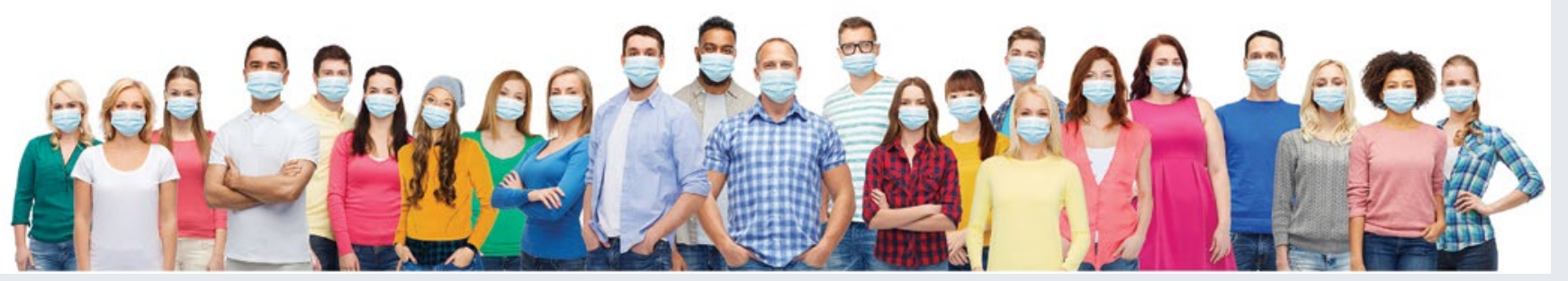

